\title{
Is HIPEC beneficial in platinum resistant relapsed ovarian cancer?
}

\author{
Nicolae BACALBASA ${ }^{1,2}$, Irina BALESCU ${ }^{3}$, Adina CROITORU ${ }^{4,5}$, Simona DIMA 4 , \\ Mihaela VILCU ${ }^{1,2}$, Iulian BREZEAN $N^{1,2}$ \\ 1"Carol Davila" University of Medicine and Pharmacy, Bucharest, Romania \\ 2"Ion Cantacuzino" Clinical Hospital, Bucharest, Romania \\ ${ }^{3}$ Ponderas Academic Hospital, Bucharest, Romania \\ ${ }^{4}$ Fundeni Clinical Institute, Bucharest, Romania \\ ${ }^{5}$ Faculty of Medicine, "Titu Maiorescu" University, Bucharest, Romania
}

\begin{abstract}
Ovarian cancer remains one of the most aggressive biological malignancies which is characterised by a high capacity of relapse even in cases in which a curative intent treatment is performed. In such cases the therapeutic options are rather limited, especially in platinum resistant lesions. The aim of this paper is to analyse the reported results after performing intraperitoneal heated chemotherapy in such cases.
\end{abstract}

Keywords: intraperitoneal heated chemotherapy (HIPEC), ovarian cancer, platinum resistance

\section{INTRODUCTION}

Ovarian cancer still represents the leading cause of death among gynaecological malignancies worldwide, especially due to the fact that most often patients remain asymptomatic for a long period of time and are diagnosed in advanced stages of the disease. In such cases peritoneum remains one of the most commonly involved organs, representing in the meantime a preferred pattern for tumoral spread. In such cases it has been widely demonstrated that one of the most important prognostic factors is represented by the completeness of cytoreduction. However, even if cytoreduction to no visible residual disease is achieved, a significant number of cases will experience recurrent disease at a certain moment. In order to impede this process various therapeutic strategies have been proposed (1-3).

\section{The principles of HIPEC}

The method consists of introducing a heated chemotherapeutic agent into the abdominal cavity after completing cytoreductive surgery, and has several benefits when compared to standard, normothermic, intravenous chemotherapy. First of all, higher dose of chemotherapeutic agents can be administrated in this manner due to the presence of the peritoneo-plasmatic barrier which impedes the absorbtion of the cytotoxic agent into the main bloodstream. In the meantime, administration of heated cytotoxic agents is associated with an improved penetration of the agent up to 3 $\mathrm{mm}$ into the tumoral cells, increasing in this way its' effect. Moreover, the procedure itself of introducing the heated cytotoxic agent is performed only after completing the debulking procedure, when no bulky residual tumor is present; in conse- 
quence, the chemotherapic agent will be able to concentrate and destroy the remnant microscopic disease (4-7). However, the procedure is an extremely demanding one and is associated with significantly higher risks of postoperative complications such as anastomotic leaks of postoperative hemoperitoneum. Therefore, it should be performed only in high specialised centers and in selected cases (7-9).

\section{Definition of platinum resistant disease}

It has been widely demonstrated that even if the principles of debulking surgery to no residual disease are successfully applied and are followed by the administration of the standard adjuvant chemotherapeutic protocol based on taxanes and platinum salts, up to $70 \%$ of patients who had been initially diagnosed in an advanced stage of the disease will develop recurrent disease at a certain point of their evolution. However, one of the most important prognostic factors at the moment when relapsed ovarian cancer is diagnosed is represented by the disease free survival interval. Therefore, patients who develop recurrent disease within the first six months after completing the adjuvant platinum based chemotherapy are defined as being platinum resistant, while those who develop the recurrent disease after a longer period of time are considered to be platinum sensitive. The moment when recurrent disease is encountered has a crucial importance, according to this parameter different therapeutic strategies being performed: while in cases considered to be platinum sensitive a novel platinum based chemotherapy is feasible, in cases with recurrent, platinum resistant disease a second line chemotherapeutic strategy should be taken in consideration (9).

\section{Studies investigating the efficacy of HIPEC in platinum resistant relapse}

The subject of HIPEC in chemo resistant ovarian cancer relapse has been widely studied so far; however, conflicting results have been reported, a standard therapeutic strategy not being established for the moment. However, the most relevant data come from the papers which compared the outcomes of platinum sensitive and platinum resistant cases.

One of the first papers which came to demonstrate the effectiveness of HIPEC in platinum resistant relapsed ovarian cancer was published by Bakrin et al. in 2012 and included 246 patients diagnosed with recurrent or persistent disease who were treated in two French centers between 1991 and 2008 (10). The authors reported a similar median survival between sensitive and resistant to platinum cases (52 months versus 48 months); the univariate analysis demonstrated that the long term outcomes were significantly influenced only by the peritoneal carcinomatosis index and by the completeness of cytoreduction. Moreover, the multivariate analysis demonstrated that when stratifying patients according to their peritoneal carcinomatosis index, cases in which the index was lower than 10 the overall prognostic was significantly influenced by the completeness of cytoreduction while in cases with a higher value of the peritoneal score, the completeness of cytoreduction did not influence the outcomes; in this latter category the only factor which seemed to influence the overall survival was represented by the performance status (10).

The data reported by the previous paper were completed one year later by the same study group which conducted a larger study in 13 French hospitals between January 1991 and December 2010. The study totalised 607 HIPEC procedures which were performed in 566 patients; among cases submitted to HIPEC for recurrent disease, there were 223 chemosensitive patients, 247 chemoresistant patients and four patients with undetermined status. When performing the survival analysis, the authors demonstrated that patients with chemoresistant lesions submitted to maximal debulking surgery and HIPEC reported a median survival of 51.6 months, while those with chemosensitive relapse experienced a median survival period of 47.2 months if complete cytoreduction was achieved; moreover, in multivariate analysis only the performance status and the peritoneal carcinomatosis index significantly impacted on the overall survival. Moreover, the authors underlined the fact that this significant benefit in terms of survival in resistant cases was achieved most often by the use of cisplatin (11). This aspect was explained through the fact that immediately after surgery, in the absence of the peritoneal adhesions and in the presence of the peritoneo-plasmatic barrier, a particular pharmacokinetic behaviour of the platinum salts is to expect (12). These data were considered to be pretty encouraging due to the fact that the median overall survival in chemotreated, platinum resistant recurrence is usually not longer than 12 months (13).

In the study conducted by Brown et al. and published in 2015 the authors included 53 patients submitted to HIPEC for recurrent ovarian cancer (14); among these cases there were 41 patients with platinum resistant disease in whom the au- 
thors used a 90 minutes perfusion of mitomycin C and 12 cases diagnosed with platinum sensitive disease in which a carboplatin/oxaliplatin perfusion was used. The median interval between the last cycle of chemotherapy and HIPEC for recurrent disease was of 42 weeks while the mean peritoneal carcinomatosis index was 19.1. The authors demonstrated that by using this method a significant benefit in terms of survival can be achieved even in heavily prechemotreated patients, irrespectively to the histopathological subtype; moreover, patients considered as being platinum resistant experienced an overall survival of 33.9 months while cases with platinum sensitive disease experienced an overall survival of 17.9 months. In the meantime the authors underlined once again the influence of the completeness of cytoreduction surgery whenever HIPEC association is performed (14).

Another interesting study conducted on this theme was published by Spiliotis et al. and was published in the Annals of Surgical Oncology in 2015 (15). The study included 120 women submitted to surgery for relapsed ovarian cancer who were randomised in two groups: the first group was submitted to debulking surgery and HIPEC followed by systemic chemotherapy while the second group was submitted to debulking surgery followed by systemic chemotherapy. The authors demonstrated a significant difference in terms of survival for the first group (26.7 months versus 13.4 months) when compared to the second group; moreover, the authors observed no difference in terms of survival among chemoresistant and chemosensitive patients from the first group (the reported median survival being of 26.6 and 26.8 months respectively). In the meantime, among patients from the second group a significant difference in terms of survival was seen among platinum resistant and platinum sensitive cases (10.2 months versus 15.2 months, $p=0.002$ ). As for the type of chemotherapic agent which was used for the HIPEC procedure, it was represented by cisplatin and paclitaxel for platinum sensitive cases and doxorubicin and placlitaxel or mitomycin for platinum resistant lesions. These data came to demonstrate once again the utility of the method even in the setting of platinum resistant disease (15).

However, in a more recent study conducted by an Italian study group, conflicting results have been reported; contrarily to the French study, the Italian one came to demonstrate the efficacy of HIPEC exclusively in treating chemosensitive relapse from ovarian cancer; in the meantime, no clear benefit in terms of survival could be demonstrated for cases presenting resistant to platinum disease (16). Moreover, other studies excluded from the very beginning patients with platinum resistant disease from their study groups which aimed to investigate the effectiveness of HIPEC in recurrent ovarian cancer (17).

\section{CONCLUSIONS}

Performing a HIPEC procedure in a patient with platinum resistant relapse remains a full of controversies issue, conflicting results being reported so far. However, the largest, randomised studies seem to clearly demonstrate the effectiveness of the procedure in terms of survival. Another intensively debated subject is represented by the chemotherapic agent who should be used; while most authors consider that in such cases other cytotoxic agents such as doxorubicin or mitomycin should be used, the French authors sustain the fact that platinum salts such as cisplatin should not be formally excluded.

\section{Acknowledgement}

This work was supported by the project entitled „Multidisciplinary Consortium for Supporting the Research Skills in Diagnosing, Treating and Identifying Predictive Factors of Malignant Gynecologic Disorders", project number PN-III-P1-1.2PCCDI2017-0833.

Conflict of interest: none declared

\section{REFERENCES}

1. Siegel RL, Miller KD, Jemal A. Cancer Statistics, 2017. CA Cancer J.Clin. 2017; 67: 7-30.

2. Ceresoli M, Verrengia A, Montori G et al. Effect of cytoreductive surgery and hyperthermic intraperitoneal chemotherapy on relapse pattern in primary epithelial ovarian cancer: a propensity score based case-control study. J.Gynecol.Oncol. 2018; 29: e53.

3. Wang $Y$, Ren F, Chen $P$ et al. Effects of CytoReductive surgery plus hyperthermic IntraPEritoneal chemotherapy (HIPEC) versus CytoReductive surgery for ovarian cancer patients: A systematic review and meta-analysis. Eur.J.Surg. Oncol. 2019; 45: 301-9.

4. Sugarbaker PH, Graves T, DeBruijn EA et al. Early postoperative intraperitoneal chemotherapy as an adjuvant therapy to surgery for peritoneal carcinomatosis from 
gastrointestinal cancer: pharmacological studies. Cancer Res. 1990; 50: 5790-4.

5. Sun X, Li XF, Russell J et al. Changes in tumor hypoxia induced by mild temperature hyperthermia as assessed by dual-tracer immunohistochemistry. Radiother.Oncol. 2008; 88: 269-76.

6. Teicher BA, Kowal CD, Kennedy KA et al. Enhancement by hyperthermia of the in vitro cytotoxicity of mitomycin C toward hypoxic tumor cells. Cancer Res. 1981; 41 : 1096-9.

7. VanderWaal R, Thampy G, Wright WD et al. Heat-induced modifications in the association of specific proteins with the nuclear matrix. Radiat.Res. 1996; 145 : 746-53.

8. Chua TC, Yan TD, Saxena A et al. Should the treatment of peritoneal carcinomatosis by cytoreductive surgery and hyperthermic intraperitoneal chemotherapy still be regarded as a highly morbid procedure?: a systematic review of morbidity and mortality. Ann.Surg. 2009; 249: 900-7.

9. Jewell A, McMahon M, Khabele D. Heated Intraperitoneal Chemotherapy in the
Management of Advanced Ovarian Cancer. Cancers.(Basel) 2018; 10.

10. Bakrin N, Cotte E, Golfier F et al. Cytoreductive surgery and hyperthermic intraperitoneal chemotherapy (HIPEC) for persistent and recurrent advanced ovarian carcinoma: a multicenter, prospective study of 246 patients. Ann.Surg. Oncol. 2012; 19: 4052-8.

11. Bakrin N, Bereder JM, Decullier E et al. Peritoneal carcinomatosis treated with cytoreductive surgery and Hyperthermic Intraperitoneal Chemotherapy (HIPEC) for advanced ovarian carcinoma: a French multicentre retrospective cohort study of 566 patients. Eur.J.Surg. Oncol. 2013; 39: 1435-43.

12. Cotte E, Passot G, Mohamed F et al. Management of peritoneal carcinomatosis from colorectal cancer: current state of practice. Cancer J. 2009; 15: 243-8.

13. Bristow RE, Puri I, Chi DS. Cytoreductive surgery for recurrent ovarian cancer: a meta-analysis. Gynecol.Oncol. 2009; 112: 265-74.
14. Brown CK, Williams S, McNutt L et al. Cytoreductive surgery (CRS) and HIPEC in recurrent platinum-resistant ovarian cancer. Gynecologic Oncology. 2015; 137: 114.

15. Spiliotis J, Halkia E, Lianos E et al.

Cytoreductive surgery and HIPEC in recurrent epithelial ovarian cancer: a prospective randomized phase III study. Ann.Surg. Oncol. 2015; 22: 1570-5.

16. Di Giorgio A, De laco P, De Simone M et al. Cytoreduction (Peritonectomy Procedures) Combined with Hyperthermic Intraperitoneal Chemotherapy (HIPEC) in Advanced Ovarian Cancer: Retrospective Italian Multicenter Observational Study of 511 Cases. Ann.Surg.Oncol. 2017; 24: 914-22.

17. Cianci S, Ronsini C, Vizzielli G et al. Cytoreductive surgery followed by HIPEC repetition for secondary ovarian cancer recurrence. Updates.Surg. 2019; 71: 389-94. 